\title{
Os desafios no diagnóstico e manejo da sepse neonatal: uma revisão narrativa
}

\author{
Challenges in the diagnosis and management of neonatal sepsis: a narrative review \\ Desafíos en el diagnóstico y manejo de la sepsis neonatal: una revisión narrativa
}

Analícia Neves Fiorentino ${ }^{1 *}$, Anderson Ricardo Cantareli da Silva ${ }^{2}$, Anna Carolina Flumignan Bucharles $^{3}$, Bruno Zardo Nunes Pereira ${ }^{4}$, Esther Pereira Borges Correia ${ }^{4}$, Guilherme Gabriel Marques $^{5}$, Karoline de Oliveira Lins Souto ${ }^{6}$, Leticia Batista Felis ${ }^{7}$, Matheus José Brito de Oliveira Lavinas $^{8}$, Bruno Cezario Costa Reis ${ }^{8}$.

\section{RESUMO}

Objetivo: Identificar desafios no diagnóstico e manejo da sepse neonatal, aprofundando-se em conceitos básicos e conduta no paciente. Revisão bibliográfica: Recém-nascidos com sepse neonatal podem apresentar sinais e sintomas inespecíficos ou sinais focais de infecção. Tendo em vista a falta de consenso acerca de um diagnóstico específico, torna-se imprescindível estar atento aos sinais clínicos, quando presentes, e solicitar exames laboratoriais. $O$ isolamento do agente causador em um local do corpo normalmente estéril, por meio de cultura, é o padrão ouro para definição de sepse neonatal. Entretanto, marcadores têm sido propostos para auxílio diagnóstico, uma vez que não existe um único teste isolado capaz de confirmar ou excluir o diagnóstico da doença com certeza. Pela inespecificidade clínica e demora de confirmação laboratorial por cultura, a terapia empírica é a mais comumente utilizada para o tratamento. Os tratamentos antimicrobianos empíricos são baseados na distinção entre sepse precoce e tardia. Considerações finais: A sepse neonatal enfrenta uma falta de consenso em relação a seu diagnóstico, exigindo atenção aos sinais clínicos, fatores de risco e laboratório. Uma variedade de marcadores e estratégias surgem com tempo, podendo contribuir na identificação precoce e para o uso empírico de antibióticos.

Palavras-chave: Diagnóstico, Conduta do tratamento medicamentoso, Sepse neonatal.

\section{ABSTRACT}

Objective: To identify challenges in the diagnosis and management of neonatal sepsis, delving into basic concepts and patient management. Review bibliographic: Newborns with neonatal sepsis may present nonspecific signs and symptoms or focal signs of infection. Given the lack of consensus on a specific diagnosis, it is essential to be aware of clinical signs, when present, and to request laboratory tests. The isolation of the causative agent in a normally sterile body site by culture is the gold standard for defining neonatal sepsis. However, several markers have been proposed to aid diagnosis, since there is no single isolated test able to confirm or exclude the diagnosis of the disease with certainty. Because of the clinical nonspecificity and the delay in laboratory confirmation by culture, empirical therapy is the most commonly used treatment. Empirical antimicrobial treatments are based on the distinction between early and late sepsis. Final considerations: Neonatal sepsis faces a lack of consensus regarding its diagnosis, requiring attention to clinical signs, risk factors, and laboratory. A variety of markers and strategies emerge over time that may contribute to early identification and empirical use of antibiotics.

Keywords: Diagnosis, Medication therapy management, Neonatal sepsis.

1 Universidade UNIDERP (UNIDERP), Campo Grande - MS. *E-mail: analicianf@gmail.com

2 Universidade do Extremo Sul Catarinense (Unesc), Criciúma - SC.

${ }^{3}$ Universidade Positivo (UP), Curitiba - PR.

${ }^{4}$ Universidade Unicesumar (UniCesumar), Maringá - PR.

5 Universidade Federal de Minas Gerais (UFMG), Belo Horizonte - MG.

${ }^{6}$ Universidade de São Paulo (USP), São Paulo - SP.

7 Universidade Paulista de Bauru (USP), Bauru - SP.

8 Universidade de Vassouras (UV), Vassouras - RJ. 


\section{RESUMEN}

Objetivo: Identificar los desafíos en diagnóstico y manejo de septicemia neonatal, centrándose en conceptos básicos y la conducta del paciente. Revisión bibliográfica: Los recién nacidos con sepsis neonatal pueden presentar signos y síntomas inespecíficos o signos focales de infección. Teniendo en cuenta falta de consenso sobre diagnóstico específico, es esencial estar atento a los signos clínicos cuando se presentan y solicitar pruebas de laboratorio. Aislamiento del agente causal en lugar del cuerpo normalmente estéril por medio de un cultivo es estándar de oro para definir sepsis neonatal. Sin embargo, se han propuesto marcadores para ayudar al diagnóstico, ya que no existe ninguna prueba aislada capaz de confirmar o excluir diagnóstico de la enfermedad con certeza. Debido la inespecificidad clínica y demora en la confirmación de laboratorio mediante cultivo, terapia empírica es más utilizada para tratamiento. El tratamiento antimicrobiano empírico se basa en distinción entre sepsis de inicio temprano y tardío. Consideraciones finales: Sepsis neonatal enfrenta falta de consenso en cuanto su diagnóstico, lo que exige prestar atención a los signos clínicos, factores de riesgo y laboratorio. Con el tiempo surgen diversos marcadores y estrategias que pueden contribuir a la identificación temprana y al uso empírico de los antibióticos.

Palabras clave: Diagnóstico, Administración del tratamiento farmacológico, Sepsis neonatal.

\section{INTRODUÇÃO}

Segundo a Organização Mundial da Saúde (OMS), a sepse neonatal é definida como uma disfunção orgânica possivelmente fatal causada por uma resposta descontrolada do hospedeiro à infecção durante o primeiro mês de vida. Pode ser classificada como precoce ou tardia, se ocorrer antes ou após 72 horas de vida, respectivamente (PROCIANOY RS e SILVEIRA RC, 2020). Essa afecção incide em aproximadamente 2000/100.000 dos recém-nascidos, possuindo mortalidade estimada entre $11 \%$ e 19\%, globalmente (FLEISCHMANN-STRUZEK C, et al, 2018).

Os fatores de risco que envolvem a sepse neonatal precoce e tardia são distintos. A precoce se associa à colonização materna por Streptococcus agalactiae, ruptura de membrana prematura ou prolongada por mais de 18 horas, presença de coriamnionite, febre materna e líquido amniótico fétido. Já na tardia, relaciona-se com fatores pós-parto como antibioticoterapia prolongada, monitoramento invasivo, prematuridade e permanência hospitalar (PROCIANOY RS e SILVEIRA RC, 2020).

As manifestações clínicas da sepse neonatal são diversas e inespecíficas, podendo ainda apresentar-se de forma assintomática. Dentre os sintomáticos, febre, hipotermia, dificuldade respiratória, letargia, irritabilidade, visceromegalias, hipotonia e convulsões são os principais sintomas encontrados (A SHAH B e PADBURY JF, 2013). Embora inúmeras manifestações fisiopatológicas tenham sido descritas, ainda há desafios quanto aos mecanismos envolvidos na sepse e quais são secundários à patologia associada (POLL TvD, et al., 2017).

Por se tratar de uma importante causa de mortalidade neonatal, o diagnóstico deve acontecer logo no início dos sintomas. As consequências para aqueles que sobrevivem incluem o risco elevado para morbidades como displasias pulmonares, alterações de desenvolvimento e sequelas neurocognitivas (HORNIK CP, et al., 2012; LIANG LD, et al., 2018).

O diagnóstico da sepse neonatal é comumente realizado por meio de exames laboratoriais, a fim de discriminar causas infecciosas de não-infecciosas. Exemplos incluem cultura de locais estéreis, como sangue, líquor e urina, não excluindo o diagnóstico em caso de negatividade dos mesmos. Os biomarcadores vêm sendo estudados como uma importante ferramenta diagnóstica neste arsenal (RUAN L, et al, 2018). Devido à inespecificidade da sepse neonatal, a terapêutica é comumente realizada com o uso de antibioticoterapia empírica quando não se conhece o agente etiológico, especialmente em Unidades de Terapia Intensiva Neonatal (UTIN) (GKENTZI D e DIMITRIOU G, 2019).

Frente aos vários desafios nas decisões de diagnóstico e tratamento da sepse neonatal, justifica-se um olhar mais atento à literatura para revisar as práticas já estabelecidas no manejo da sepse neonatal e as alternativas ainda em estudos experimentais e clínicos a respeito do cuidado de neonatos infectados e assim, diminuir a incidência de sepse, melhorando a qualidade da assistência pós-natal. O estudo objetivou identificar os desafios no diagnóstico e manejo da sepse neonatal, aprofundando-se em seus conceitos básicos e conduta no paciente acometido. 


\section{REVISÃO BIBLIOGRÁFICA}

\section{Diagnóstico}

Recém-nascidos com sepse neonatal podem apresentar sinais e sintomas inespecíficos ou sinais focais de infecção. Poucos podem ser esses sinais e sintomas, podendo variar de apneia isolada à taquicardia. Com isso, há a possibilidade de complicações, como insuficiência respiratória, choque, insuficiência renal e disfunção hepática (SHANE AL, et al., 2017).

A atenção, por parte dos profissionais da saúde, à instalação da sepse neonatal sintomática, bem como aos recém-nascidos expostos a fatores de risco, mas assintomáticos, é de fundamental importância. Tendo em vista a falta de consenso acerca de um diagnóstico específico para a patologia, torna-se imprescindível estar atento aos sinais clínicos, quando presentes, e solicitar exames laboratoriais. Tanto a sepse neonatal precoce quanto tardia possuem sintomatologias semelhantes, sendo letargia, febre, taquipneia e recusa alimentar os mais precoces. Assim, bebês que apresentarem estes sintomas devem ser triados laboratorialmente para confirmar o diagnóstico de sepse neonatal (KIM F, et al, 2020).

A clínica correlaciona-se conforme o sistema acometido. No sistema respiratório pode haver hipoxemia com necessidade de oxigênio suplementar e desconforto respiratório (taquipneia importante, batimento de asas do nariz, grunhidos, retração de fúrcula, tiragem intercostal e/ou subcostal). A afecção cardiovascular pode se apresentar como hipotensão, aumento do tempo de enchimento capilar, taquicardia e pulsos finos e diminuídos. A lesão renal frequentemente se apresenta como oligúria. Já o acometimento neurológico pode levar à irritabilidade, choro incoercível, hipo ou hipertonia muscular, convulsões, apneia, letargia, abaulamento de fontanelas e instabilidade térmica. As queixas gastrointestinais podem ser decorrentes de distensão abdominal, diarreia e anorexia. Icterícia, petéquias ou sangramentos espontâneos podem estar presentes. A pele também pode demonstrar sinais como surgimento de erupções cutâneas, vesículas, eritema e edema periarticular (KIM F, et al., 2020).

Tradicionalmente, $\mathrm{o}$ isolamento do agente causador em um local do corpo normalmente estéril, por meio de cultura, é o padrão ouro para definição de sepse neonatal. Os locais mais comumente utilizados são o sangue e o líquido cefalorraquidiano (LCR), podendo-se ainda fazer uso de urina e fluidos pleurais, articulares ou peritoneais para esse fim (SHANE AL, et al., 2017; MCGOVERN M, et al., 2020). Entretanto, a ausência de crescimento em meio de cultura não exclui o diagnóstico, visto que hemocultura positiva ocorre apenas em até $51 \%$ dos casos (MCGOVERN M, et al., 2020; FLEISCHMANN-STRUZEK C, et al., 2018). É válido ressaltar que, embora idealmente a obtenção de material para cultura deva ser realizada antes do início da antibioticoterapia, a mesma não deve ser atrasada em prol da realização do exame, quando indisponível (GKENTZI D e DIMITRIOU G, 2019).

Devido à demora para o crescimento da cultura (48 a 72 horas), outros exames são utilizados para corroborar com o diagnóstico do quadro, são eles: hemograma completo, contagem absoluta de neutrófilos (ANC), relação de ANC com a produção de neutrófilos imaturos, contagem de plaquetas e a proteína $C$ reativa (PCR) (KHAN F, 2019). Entretanto, diversos marcadores têm sido propostos para o auxílio diagnóstico, uma vez que não existe um único teste isolado capaz de confirmar ou excluir o diagnóstico da doença com total certeza (GKENTZI D e DIMITRIOU G, 2019).

\section{Hemograma}

Mesmo com baixo valor preditivo positivo e negativo, o hemograma completo seriado é capaz de descartar a presença da sepse. Apesar da baixa sensibilidade (29\%), leucócitos inferiores a $5000-7500 \mathrm{~mm}^{3}$ têm sido muito utilizados, uma vez que possuem alta especificidade (91\%) para o diagnóstico de sepse neonatal. Além do mais, a relação da ANC com a produção de neutrófilos imaturos têm alto valor para exclusão diagnóstica, pois uma relação maior que 0,2 é associada à sepse neonatal. A leucopenia associada a baixa ANC e a altos níveis de neutrófilos imaturos foram correlacionadas a maiores chances de infecções, sendo considerados marcadores específicos e de baixo valor preditivo negativo (SHARMA D, et al., 2017).

\section{Proteína $\mathrm{C}$ reativa}

A PCR é o teste mais utilizado para o diagnóstico de sepse neonatal. Apesar de demorar cerca de 10 a 12 horas para se elevar, sua sensibilidade aumenta entre 24 a 48 horas após o aparecimento dos sintomas, 
sendo um ótimo indicador de ausência de sepse devido ao seu valor preditivo negativo de 99\% (SHARMA D, et al., 2017; BERSANI I, et al., 2014). Embora estudos tenham demonstrado que o teste possua grande variabilidade na sensibilidade (30-80\%) e especificidade (83-100\%) (SHARMA D, et al., 2017), em conjunto com outros marcadores, como interleucina-6 (IL-6) e 8 (IL-8), procalcitonina (PCT) e CD11b e CD64, sua sensibilidade passa para $90 \%$. Ainda, se realizada desde o início do quadro, a PCR é um excelente exame de monitorização da resposta ao tratamento e possíveis complicações decorrentes da falha medicamentosa (MEMAR MY, et al., 2017).

Além da PCR convencional, a proteína C reativa de alta sensibilidade (hs-PCR) demonstrou maior sensibilidade devido aos seus menores valores de corte. Um estudo recente conseguiu delimitar os valores obtidos em: sem risco, baixo risco, risco habitual e alto risco de infecção. Entretanto, mais estudos são necessários para poder firmar seu emprego como método diagnóstico (SHARMA D et al., 2017; CHAUHAN N, et al., 2017).

\section{Procalcitonina}

A Procalcitonina (PCT) está relacionada com a imunomodulação e com a resposta vascular na Síndrome da Resposta Inflamatória Sistêmica (SIRS), especialmente por causas bacterianas. Possui um rápido aumento após 2 a 4 horas de exposição às endotoxinas bacterianas, alcançando seu pico com 6 horas e persistindo por aproximadamente 24 horas. Dessa forma, torna-se um bom marcador para o diagnóstico precoce quando comparada à PCR em infecções bacterianas (SHARMA D, et al., 2017; MEMAR MY, et al., 2017; HEDEGAARD SS, et al., 2014).

Além disso, da mesma forma que a PCR, a PCT também é um bom marcador da eficácia do tratamento e seu prognóstico (SHARMA D, et al., 2017; MEMAR MY, et al. 2017; HEDEGAARD SS, et al., 2014). Embora tenha maior sensibilidade e especificidade sozinha ( $92 \%$ e $97 \%$, respectivamente), se dosada em conjunto com a PCR, a sensibilidade e o valor preditivo negativo também aumentam (MEMAR MY et al., 2017; SHAH BA e PADBURY JF, 2014; HE Y, et al., 2019).

Entretanto, a elevação de PCT pode ter outras causas que podem ser encontradas em condições como o período de até 48 horas pós-parto, neonatos prematuros, hemorragia intracraniana, asfixia neonatal, hipoxemia neonatal, ruptura prolongada das membranas, uso de antibioticoterapia pós natal e entre outros (RUAN L, et al., 2018). Desse modo, faz-se necessário aguardar 48 horas do início da sintomatologia para avaliá-lo como um marcador confiável em vigência dessas situações (MEMAR MY, et al., 2017).

\section{Amiloide A sérica}

A amiloide A sérica (SAA), uma apolipoproteína de fase aguda, assim como PCT e PCR, tem sido reportada como um marcador prognóstico mais precoce. Esse marcador aumenta gradualmente seus níveis entre 8 a 24 horas, antes mesmo da PCR, declinando após esse período. Concentrações de $10 \mathrm{mcg} / \mathrm{mL}$ possuem sensibilidade e predição negativa de 97 a 99\%. Além disso, quando associada à PCR e à contagem de plaquetas pode ter grande valor para avaliação prognóstica de sepse tardia em pré-termos (SHARMA D, et al., 2017; HINCU MA, et al., 2020).

\section{Proteína de ligação a lipopolissacarídeos (LPB)}

A proteína de ligação a lipopolissacarídeos (LPB) é originada nos hepatócitos, células epiteliais e musculares, tendo grande valor em casos de infecções por bactérias gram-negativas. Seus níveis aumentam rapidamente com apenas 6 a 8 horas de infecção, sofrendo ainda menos alterações fisiológicas e obstétricas nos primeiros dois dias de vida. Dessa forma, é descrito como um marcador com alta sensibilidade e valor preditivo negativo especialmente na sepse neonatal precoce (SHARMA D, et al., 2017).

\section{Citocinas}

Nos últimos anos, as citocinas têm ganhado espaço como marcadores de sepse neonatal (CAMPOS DP, et al., 2010). O uso da IL-6 é proposto para o diagnóstico precoce da sepse neonatal pelo seu rápido aumento após o início da doença, antecedendo os níveis da PCR. A IL-6 isolada (menor que $130 \mathrm{pg} / \mathrm{ml}$ ) apresentou $100 \%$ de sensibilidade e $80 \%$ de especificidade para exclusão de sepse neonatal (SHARMA D, et al., 2017; 
RASHWAN NI, et al., 2017). Os níveis elevados de IL-8 e de fator de necrose tumoral $\alpha$ (TNF- $\alpha$ ) têm sido propostos tanto como marcadores da infecção quanto indicativos da gravidade da doença (SHARMA D, et al., 2017; CAMPOS DP, et al., 2010; SHAH BA e PADBURY JF, 2014).

A sensibilidade e a especificidade do TNF- $\alpha$ são $75 \%$ e $88 \%$, nessa ordem (SHAH BA e PADBURY JF, 2014). Já a IL-8 possui sensibilidade e especificidade variando de 80 a $91 \%$ e 76 a $100 \%$, respectivamente, no diagnóstico de sepse neonatal (SHARMA D, et al., 2017). A combinação desses marcadores pode apresentar sensibilidade de até $98,5 \%$, aumentando a possibilidade de diagnóstico precoce (SHARMA D, et al., 2017; CAMPOS DP, et al., 2010; DELANGHE JR e SPEECKAERT MM, 2014).

\section{Outros marcadores imunológicos}

A proteína CD163 demonstrou ser o marcador mais poderoso para diferenciar os neonatos infectados dos não infectados, possuindo sensibilidade de $100 \%$ e especificidade de $88 \%$ para o diagnóstico da infecção antes do uso de antibióticos (SHARMA D, et al., 2017).

A presepsina foi indicada como um marcador de grande especificidade e sensibilidade, podendo até ser utilizada isoladamente para detecção do quadro. Também pode ser empregada na monitorização das intervenções terapêuticas frente à nova obtenção de culturas, visto que já no primeiro dia de tratamento seus níveis apresentam declínio, fato que não ocorre com a PCR e a PCT (RUAN L et al., 2018; TZIALLA C, et al., 2018).

\section{Resumo dos marcadores da sepse}

Os principais marcadores para sepse neonatal são, portanto, o leucograma, capaz de descartar a presença de sepse; a PCR, um dos testes mais comumente utilizados tanto para diagnóstico quanto para monitoramento da resposta antibiótica (SHARMA D et al., 2017); a PCT, que possui maior sensibilidade se comparada à PCR, porém é necessário aguardar uma janela de 48 horas para julgar seus valores e ser utilizada com confiança (SHARMA D, et al., 2017; MEMAR MY, et al., 2017); a IL-6, IL-8 e TNF- $\alpha$, que quando combinados passam a apresentar uma sensibilidade de até 98,5\%(SHARMA D, et al., 2017; CAMPOS DP, et al., 2010; DELANGHE JR e SPEECKAERT MM, 2014); e a CD163, um excelente marcador para detectar e diferenciar os neonatos infectados dos não infectados, além de possivelmente antecipar o diagnóstico de sepse neonatal antes mesmo do início da antibioticoterapia empírica, podendo prevenir seu uso desnecessário (SHARMA D, et al., 2017).

\section{Manejo do paciente com sepse neonatal}

Pela inespecificidade clínica e demora de confirmação laboratorial por cultura, a terapia empírica é a mais comumente utilizada para o tratamento da sepse neonatal. Os tratamentos antimicrobianos empíricos propostos são baseados na distinção entre sepse precoce e tardia. Quando o resultado da cultura estiver disponível e o patógeno e sua sensibilidade forem relatados, deve-se adaptar imediatamente o antimicrobiano (GKENTZI D e DIMITRIOU G, 2019).

\section{Sepse precoce}

Os regimes mais utilizados são uma combinação de ampicilina e gentamicina, uma vez que Streptococcus do grupo B e Enterobacteriaceae são os agentes causais mais comuns de sepse precoce em todo o mundo, visto que estão presentes na flora do trato genitourinário e gastrointestinal materna (GKENTZI D e DIMITRIOU G, 2019).

\section{Sepse tardia}

Recomenda-se o uso de penicilina semissintética combinada com um aminoglicosídeo. Em alternativa, pode-se utilizar piperacilina e tazobactam na combinação para o tratamento de infecções suspeitas por patógenos Gram-negativos. Em caso de ausência de meningite, o uso de cefalosporina deve ser evitado (GKENTZI D e DIMITRIOU G, 2019).

A sepse tardia também pode necessitar de cobertura para fungos, especialmente para Candida. $O$ tratamento de primeira linha é desoxicolato de anfotericina $B$, podendo ser utilizado em alternativa 0 
fluconazol. Caso o trato urinário esteja envolvido, a anfotericina b lipossomal pode ser administrada. Contudo, o tratamento antifúngico deve ser interrompido se as hemoculturas vierem negativas para esses patógenos (GKENTZI D e DIMITRIOU G, 2019).

Embora os Staphylococcus aureus resistentes à meticilina (MRSA) sejam prevalentes nos casos de sepse neonatal tardia, a vancomicina deve ser evitada como tratamento inicial, visto que seu uso indiscriminado é importante fator para o surgimento de floras multirresistentes e infecções fúngicas invasivas. Entretanto, devido à taxa variável de resistência dos estafilococos coagulase-negativos (ECN), a vancomicina ainda é muito utilizada em UTINs (PROCIANOY RS e SILVEIRA RC, 2019).

Alguns serviços optam por dar início ao tratamento com oxacilina, devendo escalonar o uso com vancomicina em 48 horas na ausência de melhora ou piora clínica, visto que não aumenta a mortalidade, duração do quadro ou taxa de complicações (PROCIANOY RS e SILVEIRA RC, 2019). Algumas UTINs propõem seu uso somente após isolamento do patógeno em hemocultura, e não como terapia empírica (GKENTZI D e DIMITRIOU G, 2019).

A fim de diminuir o uso de antibioticoterapia desnecessária, um grupo de pesquisadores desenvolveu uma calculadora para recém-nascidos com mais de 34 semanas que leva em consideração os fatores de risco, como tempo de ruptura da membrana amniótica, presença de colonização por estreptococo $B$ e idade gestacional, e a clínica do paciente, sendo capaz de predizer a probabilidade de sepse precoce. Sua aplicabilidade foi capaz de reduzir o uso de antibióticos em $40 \%$, sem causar aumento no risco de resultados falso negativos. Além disso, na Europa, alguns centros tentaram diminuir o emprego de antibioticoterapia ao dar mais valor à observação clínica versus a requisição de exames laboratoriais exaustivos e seriados, diminuindo o tempo de estadia hospitalar e, consequentemente, diminuindo a taxa de sepse neonatal tardia (PROCIANOY RS e SILVEIRA RC, 2019).

\section{Profilaxia de sepse neonatal}

Conhecendo os fatores de risco, o Centro de Controle e Prevenção de Doenças (CDC) dos Estados Unidos recomenda os seguintes cuidados para evitar a sepse neonatal: triagem universal de gestantes para colonização estreptocócica com 34 a 37 semanas e fazer antibioticoprofilaxia quando detectado, durante o trabalho de parto ou após ruptura da membrana amniótica. Caso o rastreio seja desconhecido, a quimioprofilaxia com penicilina cristalina será realizada na presença de febre intraparto, tempo de amniorrexe maior que 18 horas e prematuridade (PROCIANOY RS e SILVEIRA RC, 2019).

\section{Choque séptico}

A Campanha de Sobrevivência a Sepse de 2020 propôs medidas iniciais que devem ser feitas na vigência de choque séptico: (1) estabilização inicial, visando padronizar os cuidados e melhorar os desfechos; (2) fluidoterapia com cristalóide, feita conforme o quadro do paciente, categorizando-o em hipotensivo ou não e podendo empregar drogas vasoativas caso refratariedade às medidas tomadas; e (3) monitorização clínica e dos parâmetros hemodinâmicos (WEISS SL, et al., 2020).

O suporte ventilatório pode ser indicado, sendo ele invasivo ou não, conforme a necessidade do quadro. É importante ressaltar que Etomidato é proscrito, uma vez que estudos recentes demonstraram maior mortalidade em um grupo com choque ou sepse meningocócica quando comparado àqueles que fizeram outra combinação de sedativos. Também foi reportado associação forte entre etomidato e insuficiência adrenal. Caso seja necessário realizar uma intubação de sequência rápida, baixas doses de benzodiazepínicos e cetamina ou atropina são indicadas (WEISS SL, et al., 2020).

O uso de corticoterapia adjuvante permanece sendo controverso, mas em casos de choque resistente à catecolaminas e com risco absoluto de insuficiência adrenal, a hidrocortisona pode ser utilizada. A utilização de Insulinoterapia pode ser indicada com segurança para o controle glicêmico, tendo como alvo uma glicemia menor ou igual a $140 \mathrm{mg} / \mathrm{dL}$ (WEISS SL, et al., 2020).

A nutrição enteral deverá ser iniciada através de sonda já nas primeiras 48 horas na ausência de contraindicações formais. Caso a escolha da via seja parenteral, deve-se limitar seu uso a até 7 dias. Além disso, não existe indicação para realização de transfusão profilática de plaquetas e plasma, logo, não deve 
ser feita tomando como base apenas uma plaquetopenia na ausência de sangramentos, discrasias sanguíneas ou trombocitopenia. Já a transfusão de hemácias, deverá ser realizada caso hemoglobina $<7$ g/dL (WEISS SL, et al., 2020).

\section{CONSIDERAÇÕES FINAIS}

Patologia prevalente e de marcante morbimortalidade, a sepse neonatal enfrenta uma falta de consenso em relação a seu diagnóstico, exigindo atenção aos sinais clínicos, fatores de risco e laboratório. Ademais, uma extensa variedade de marcadores e estratégias diagnósticas surgem com o passar do tempo, podendo contribuir na identificação precoce e no desenvolvimento de sistemas para o uso empírico de antibióticos, otimizando o manejo das diferentes formas de sepse e também diminuindo os transtornos gerados pela doença. $O$ estudo revisa e categoriza o amplo conhecimento sobre o assunto, indicando a aplicabilidade das formas de diagnóstico e tratamento. Contudo, a introdução desses métodos ainda enfrenta problemas acerca de disponibilidade e ausência de protocolos específicos nos serviços de saúde.

\section{REFERÊNCIAS}

1. BERSANI I, et al. Use of Early Biomarkers in Neonatal Brain Damage and Sepsis: State of the Art and Future Perspectives. Biomed research international, 2015; 2015:1-10.

2. CAMPOS DP, et al. Sepse neonatal precoce: níveis de citocinas no sangue de cordão umbilical no diagnóstico e durante o tratamento. Jornal de Pediatria [online], 2010; 86(6): 509-514.

3. CHAUHAN N, et al. Potential biomarkers for effective screening of neonatal sepsis infections: An overview. Microbial Pathogenesis, 2017; 107:234-242.

4. DELANGHE JR, SPEECKAERT MM. Translational research and biomarkers in neonatal sepsis. Clinica Chimica Acta, $2015 ; 451(\mathrm{Pt} \mathrm{A}):$ 46-64.

5. FLEISCHMANN-STRUZEK C, et al. The global burden of paediatric and neonatal sepsis. The Lancet Respiratory Medicine, 2018; 6(3): 223-230.

6. GKENTZI D, DIMITRIOU G. Antimicrobial Stewardship in the Neonatal Intensive Care Unit: An Update. Current Pediatric Reviews, 2019; 15(1): 47-52.

7. HE Y, et al. Efficacy and safety of applying a neonatal early-onset sepsis risk calculator in China. Journal of Paediatrics and Child Health, 2020; 56(2): 237-243.

8. HEDEGAARD SS, et al. Diagnostic utility of biomarkers for neonatal sepsis - a systematic review. Infectious diseases, 2015; 47(3): 117-24.

9. HINCU MA, et al. Relevance of Biomarkers Currently in Use or Research for Practical Diagnosis Approach of Neonatal Early-Onset Sepsis. Children (Basel), 2020; 7(12): 309.

10. HORNIK CP, et al. Early and late onset sepsis in very-low-birth-weight infants from a large group of neonatal intensive care units. Early Human Development, 2012; 88(2): 69-74.

11. KHAN F. C-reactive Protein as a Screening Biomarker in Neonatal Sepsis. Journal of College of Physicians and Surgeons Pakistan (JCPSP), 2019; 29(10): 951-953.

12. KIM F, et al. Neonatal sepsis. BMJ, 2020; $1: 371: \mathrm{m} 3672$.

13. LIANG LD, et al. Predictors of Mortality in Neonates and Infants Hospitalized With Sepsis or Serious Infections in Developing Countries: a systematic review. Frontiers In Pediatrics, 2018; 6: 1-12.

14. MCGOVERN M, et al. Challenges in developing a consensus definition of neonatal sepsis. International Pediatric Research Foundation, 2020; 88(1): 14-26.

15. MEMAR MY, et al. Immunologic biomarkers for diagnostic of Early-Onset Neonatal Sepsis. The Journal of MaternalFetal and Neonatal Medicine, 2019; 32(1): 143-153.

16. POLL TVD, et al. The immunopathology of sepsis and potential therapeutic targets. Nature Reviews Immunology, 2017; $17(7): 407-420$

17. PROCIANOY RS, SILVEIRA RC. The challenges of neonatal sepsis management. Jornal de Pediatria, 2020; 96(1): 80-86.

18. RASHWAN NI, et al. Validity of Biomarkers in Screening for Neonatal Sepsis - A Single-Center Hospital based Study. Pediatrics and Neonatology, 2019; 60(2): 149-155.

19. RUAN L, et al. The combination of procalcitonin and C-reactive protein or presepsin alone improves the accuracy of diagnosis of neonatal sepsis: a meta-analysis and systematic review. Critical Care, 2018; 22(1):316.

20. SHANE AL, et al. Neonatal sepsis. The Lancet, 2017; 390(10104): 1770-1780.

21. SHAH BA, PADBURY JF. Neonatal sepsis: an old problem with new insights. Virulence, $2014 ; 5(1): 170-8$.

22. SHARMA D, et al. Biomarkers for diagnosis of neonatal sepsis: a literature review. The Journal of Maternal-Fetal and Neonatal Medicine, 2017; 31(12): 1646-1659.

23. TZIALLA C, et al. New Diagnostic Possibilities for Neonatal Sepsis. American Journal of Perinatology, 2018; 35(6): 575-577.

24. WEISS SL, et al. Surviving sepsis campaign international guidelines for the management of septic shock and sepsisassociated organ dysfunction in children. Pediatric Critical Care Medicine, 2020; 21(2): e52-e106. 